\title{
Exercise Parameters Predict Technique Survival in Patients on Peritoneal Dialysis
}

\author{
Takashin Nakayama ${ }^{a}$ Kiyotaka Uchiyama ${ }^{a}$ Naoki Naoki ${ }^{a, b}$ \\ Kohkichi Morimoto $^{a}$ Kaori Muraoka $^{c}$ Keika Adachi ${ }^{a}$ Takahiro Kasai $^{b}$ \\ Kazutoshi Miyashita $^{\text {a }}$ Shu Wakino ${ }^{\text {a }}$ Hiroshi Itoh $^{a}$ \\ aDivision of Endocrinology, Metabolism and Nephrology Department of Internal Medicine, Keio University \\ School of Medicine, Hanamachi, Shinjuku-ku, Japan; 'bepartment of Nephrology, International University of \\ Health and Welfare School of Medicine, Chiba, Japan; 'Department of Rehabilitation Medicine, Keio University \\ School of Medicine, Shinjuku-ku, Japan
}

\section{Keywords}

Aerobic capacity · Exercise capacity · Incremental shuttle walking test - Assisted peritoneal dialysis - Peritoneal dialysis-related outcome · Frailty

\footnotetext{
Abstract

Objective: The incremental shuttle walking test (ISWT) is an important marker of aerobic capacity in patients on peritoneal dialysis (PD). This study aimed to evaluate its predictive value for PD-related outcomes. Methods: This single-center cohort study recruited outpatients on maintenance PD from our hospital between March 2017 and March 2018. Exercise capacity was assessed using measurement of ISWT and handgrip and quadriceps strength. Patients were divided into 2 groups according to the median of exercise capacity and prospectively followed up until cessation of PD, death, or the study end (October 2019). The primary end point of this study was technique survival rate, and secondary out-
}

comes were rates of peritonitis-free survival and PD-related hospitalization-free survival. Results: Among the 50 participants, age and PD vintage were [median (IQR)] 62.5 (58.3-70) and 3.5 (1.3-6.5) years, respectively. At the end of the study, 3 of the 28 participants (11\%) in the long-ISWT group and 13 of the 22 participants (59\%) in the short-ISWT group were transferred to hemodialysis. The short-ISWT group showed lower technique survival rate $(p<0.001)$, peritonitis-free survival rate $(p=0.01)$, and PD-related hospitalization-free survival rate $(p<0.01)$ than the long-ISWT group, whereas those survival rates did not differ when participants were divided by handgrip or quadriceps strength. Multivariate analysis revealed lower ISWT to be independently associated with technique failure $(p=0.002)$. Conclusion: The ISWT is an important predictor of technique survival for patients on PD. Monitoring and enhancing ISWT as a marker of aerobic capacity might improve PD-related outcomes.

(c) 2020 The Author(s)

Published by S. Karger AG, Basel karger@karger.com www.karger.com/bpu

Karger $\stackrel{\text { ' }}{5}$

BOPEN ACCESS
(C) 2020 The Author(s)

Published by S. Karger AG, Basel

This article is licensed under the Creative Commons AttributionNonCommercial-NoDerivatives 4.0 International License (CC BYNC-ND) (http://www.karger.com/Services/OpenAccessLicense). Usage and distribution for commercial purposes as well as any distribution of modified material requires written permission.
Kiyotaka Uchiyama

Division of Endocrinology, Metabolism and Nephrology Department of Internal Medicine, Keio University School of Medicine

35, Shinanomachi, Shinjuku-ku 160-8582 (Japan)

kiyo.0817.piyo@gmail.com 


\section{Introduction}

One of the relevant problems on peritoneal dialysis (PD) treatment is the higher technique failure rate than hemodialysis (HD), leading to the low penetration rate or the severe complications including congestive heart failure and peritonitis [1-3]. Therefore, identification of the risk factors for PD technique failure and research into potential interventions are of high importance.

Declined exercise capacity is a significant problem for patients with CKD, especially those undergoing dialysis, although the etiology is multifactorial and remains unclear [4]. Uremic sarcopenia is defined as reduced exercise capacity and skeletal muscle mass and has been reported to be independently associated with a high risk of mortality in CKD patients including those undergoing PD $[5,6]$. Furthermore, it has been reported that a low score for the 6-min walk distance is an independent risk factor for mortality among patients undergoing PD [7], and previous cross-sectional study demonstrated the association of quality of life with exercise capacity in these patients [8]. Thus, exercise capacity is the focus of a considerable amount of research attention in the field of $\mathrm{PD}$, but the contribution to technique survival rate is still uncertain.

The present study is a prospective cohort study involving outpatients undergoing PD in our hospital with the aim of determining the contribution of exercise tolerance to PD-related outcomes including technique survival time, peritonitis-free survival time, and PD-related hospitalization-free survival time in patients undergoing PD. Furthermore, we hypothesized that assisted PD might improve technique survival time in patients with decreased exercise capacity, and recorded and included the presence of assisted PD into the analysis.

\section{Materials and Methods}

\section{Study Population}

This study and all its protocols were reviewed and approved by the Ethics Committee of our hospital, and written informed consent was obtained from all patients prior to participation. We recruited all stable patients aged 20-90 years who received PD for 3 months or more in our hospital between March 2017 and March 2018 for the present single-center cohort study. Because the participants were required to undergo exercise testing at the baseline, the following exclusion criteria were set based on the guidelines of the American College of Sports Medicine and Japanese Circulation Society [9, 10]: uncontrolled hypertension (blood pressure $>180 / 110 \mathrm{~mm} \mathrm{Hg}$ ); severe anemia (hemoglobin $<7 \mathrm{mg} / \mathrm{dL}$ ); active and proliferative diabetic retinopathy; symptomatic coronary artery disease or cerebrovascular disease within 3 months prior to study recruitment; current heart failure (New York Heart Association class III or IV); symptomatic and fatal arrhythmia; significant valvular heart disease; and difficulty walking without an aid due to orthopedic problems, history of cerebrovascular disease, or severe peripheral artery disease.

\section{Data Collection and Participants Evaluation}

The following demographic data were collected at the time of enrollment from medical records: age, sex, PD vintage (years), modality of PD (continuous ambulatory or automated PD), diabetes mellitus (DM), smoking status (smoker [current or ex-smoker] or nonsmoker), history of cardiovascular disease, and the presence of assisted PD. The Charlson Comorbidity Index (CCI) was calculated from the records. Exercise parameters, patients' frailty, blood pressure, and anthropometric data including BMI $\left(\mathrm{kg} / \mathrm{m}^{2}\right)$ or skeletal mass index (SMI) $\left(\mathrm{kg} / \mathrm{m}^{2}\right)$ were measured as previously described $[6,8]$. Briefly, exercise testing was carried out by assessing the incremental shuttle walking test (ISWT) and handgrip and quadriceps strength. The total distance of ISWT, which allows the patients to walk between 2 cones at 10 -m intervals, setting a pace with a beeper, and gradually increasing the speed of the beeper until the patient is unable to keep up with the set pace or the patient stops due to fatigue, and highest values of 2 measurements of handgrip and quadriceps strength of both hands and legs, respectively, were used for further analysis. All exercise assessments were carried out by the same rehabilitation doctor and measured with the abdomen free of dialysis fluid.

\section{Biochemical Analyses}

The following biochemical data were obtained at the time of enrollment. Parameters of dialysis adequacy including urea kinetics $(\mathrm{Kt} / \mathrm{V})$ and urine output were determined using 24-h urine and PD-fluid collections. Urine output was also recorded. Serum albumin $(\mathrm{mg} / \mathrm{dL})$, hemoglobin $(\mathrm{mg} / \mathrm{dL})$, calcium $(\mathrm{mg} / \mathrm{dL})$, phosphate $(\mathrm{mg} / \mathrm{dL})$, intact parathyroid hormone $(\mathrm{pg} / \mathrm{mL}), \mathrm{C}$-reactive protein $(\mathrm{mg} / \mathrm{L})$, and human atrial natriuretic peptide $(\mathrm{pg} / \mathrm{mL})$ levels were measured using blood samples. Geriatric nutritional risk index (GNRI) was calculated from patients' body weight and serum albumin level [11].

\section{Follow-Up}

Participants were divided into 2 groups according to the median of exercise capacity including ISWT and handgrip and quadriceps strength. Namely, long- and short-ISWT groups were defined as ISWT of $\geq 290 \mathrm{~m}$ and $<290 \mathrm{~m}$; high- and low-handgripstrength groups were defined as having a handgrip strength of $\geq 28.5 \mathrm{~kg}$ and $<28.5 \mathrm{~kg}$; and high- and low-quadriceps-strength groups were defined as having quadriceps strength of $\geq 22.3 \mathrm{~kg}$ and $<22.3 \mathrm{~kg}$, respectively. All participants were prospectively followed up until the cessation of PD, death, or the end of the study (October 2019). Reasons for cessation of PD and causes of peritonitis were also recorded, along with the relevant dates. The primary end point of this study was technique survival time, which is equivalent to survival free of unintended transition to HD. Participants who died or elected to change to HD or transplantation were censored as previously mentioned in recommendation by International Society for Peritoneal Dialysis [12]. A sensitivity analysis in which death and transplantation were regarded as technique failure was also performed. Secondary outcomes were peritonitis-free survival time and $\mathrm{PD}$-related hospitalization-free 
Fig. 1. Flowchart of study enrollment. PD, peritoneal dialysis.

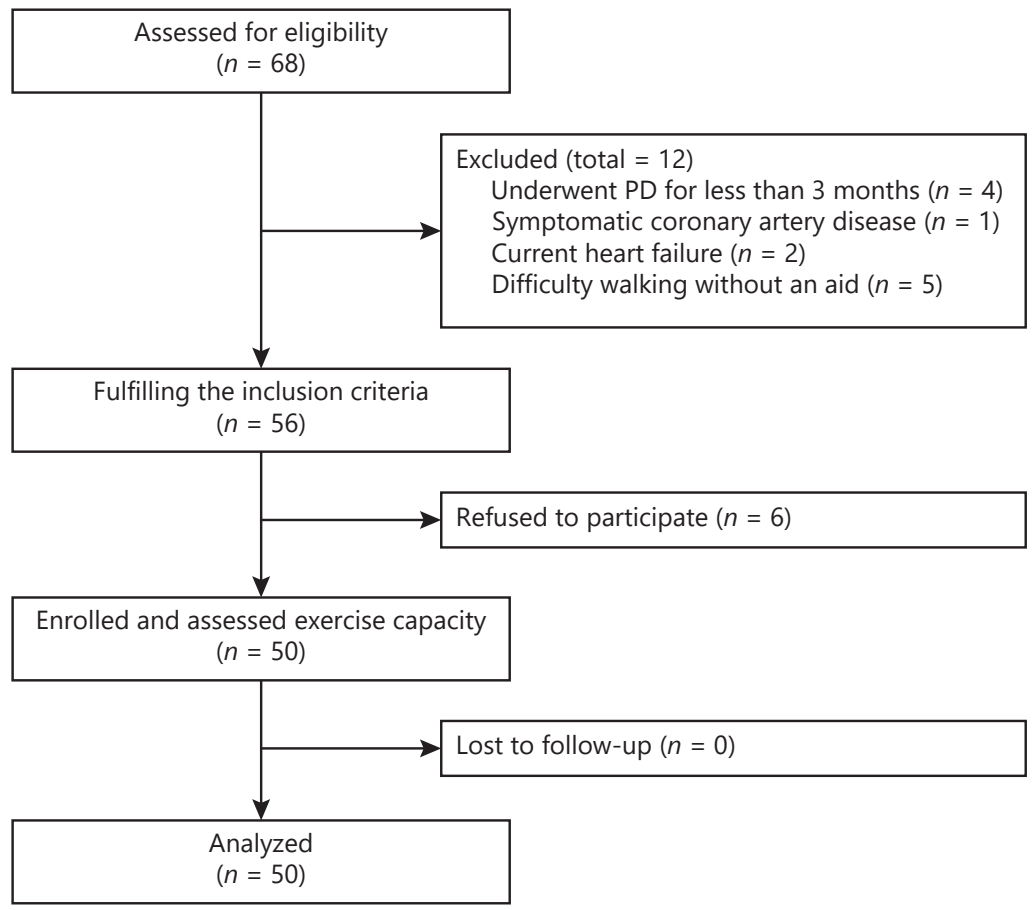

survival time. All outcomes, including technique failure, peritonitis, and PD-related hospitalization, were evaluated simultaneously at the end of the study.

\section{Statistical Analyses}

Continuous variables are expressed as means \pm standard deviation or median (25th-75th percentile) according to normality tested by Shapiro-Wilk test and binary variables as percentages. The unpaired Student's $t$ test (continuous variables with normal distribution), Mann-Whitney U test (continuous variables without normal distribution), and $\chi^{2}$ test (binary variables) were used to compare parameters between the groups. Survival curves were plotted using the Kaplan-Meier method and compared using log-rank tests. A crude or adjusted Cox proportional hazard model was used to evaluate the hazard ratio and $95 \%$ confidence interval for technique survival. Because the aim of this study was to exploratory clarify the association between exercise capacity and technique survival in patients undergoing PD, the parameters of exercise capacity that were identified to be significant predictors of technique failure in univariate Cox analysis were entered into the multivariate regression model. Moreover, variables that have been previously reported to be associated with technical survival were included, independent of the association with technique failure in the univariate model in the present study. Age, DM, use of assisted $\mathrm{PD}$, frailty, CCI, PD vintage, urine output, GNRI, and ISWT were included as candidate independent variables. However, because strong and significant correlations were observed between DM and CCI and between PD vintage and urine output, DM and urine output were consequently excluded as independent variables, tak- ing multicollinearity into consideration [13-15]. Multivariate model using all these independent parameters (model 1) was followed by a stepwise backward analysis, in which variables were subsequently excluded from the model to create a final model that only contained variables that were significantly associated with technique failure (model 2) [8]. Moreover, because there were competing risk events against technique failure, including death and transplantation, the subdistribution hazard model proposed by Fine and Gray was used for the multivariate analysis in addition to the cause-specific hazard model for standard Cox regression $[16,17]$.

\section{Results}

\section{Clinical Characteristics}

The source population comprised 68 patients, 56 of whom were eligible (Fig. 1). Eight patients were excluded due to symptomatic coronary artery disease $(n=1)$, current heart failure $(n=2)$, and difficulty walking without an aid $(n=5)$. Among these 8 patients, 2 patients undergoing self-care were transferred to HD in 12.8 and 13.7 months and 6 patients with assisted PD died without a modality change in $16.0 \pm 9.7$ months. Additionally, 4 patients who underwent PD for less than 3 months were considered as unstable and were excluded as candidates 
Table 1. Baseline characteristics of the study population and of the groups divided according to the median of ISWT

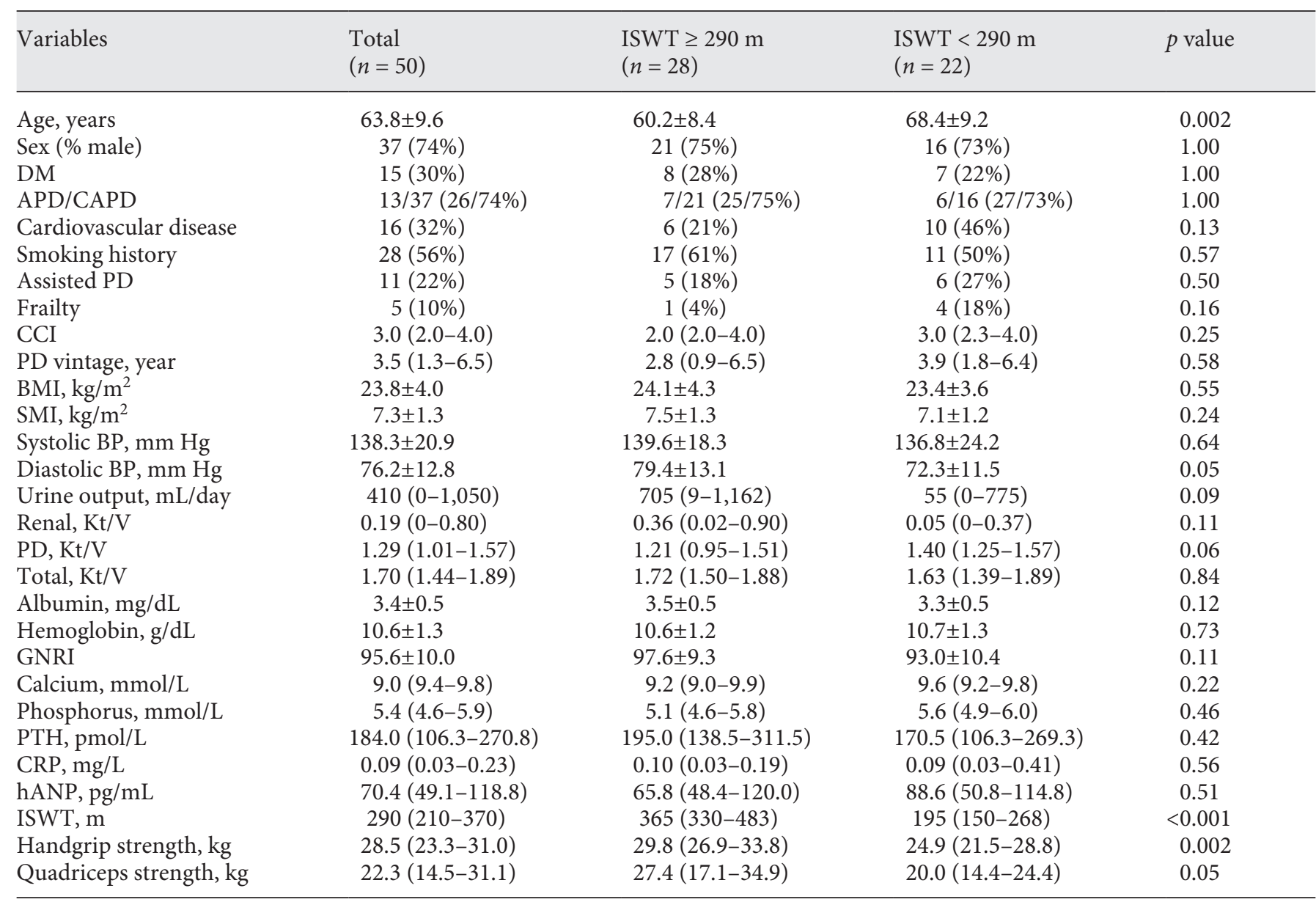

Continuous variables are expressed as means \pm standard deviation or median (25th-75th percentile) and binary variables as number (percentage). ISWT, incremental shuttle walking test; HD, hemodialysis; APD, automated peritoneal dialysis; CAPD, continuous ambulatory peritoneal dialysis; SMI, skeletal mass index; BP, blood pressure; GNRI, geriatric nutritional risk index; PTH, parathyroid hormone; CRP, C-reactive protein; hANP, human atrial natriuretic peptide; CCI, Charlson Comorbidity Index; PD, peritoneal dialysis.

for baseline assessment. Three of the 4 patients continued PD until the end of study and the remaining 1 patient underwent kidney transplantation. Eventually, 50 patients who provided consent to participate in the study were enrolled. The baseline PD vintage of the study cohort was 3.5 (1.3-6.5) years; $16(32 \%)$ patients discontinued PD and were transferred to HD during the study period. The cause of transfer was peritonitis $(n=8)$, difficulties performing PD due to decreased activities of daily living $(n=3)$ or cerebrovascular disease leading to physical disability $(n=2)$, catheter malfunction $(n=1)$, major abdominal surgery $(n=1)$, and difficulty in controlling hypertension with volume overload $(n=1)$. One participant underwent kidney transplantation, and 2 died without changing PD modality. No patients were transferred to HD in a planned fashion. The median follow-up period was $27.5(13.0-30.6)$ months, and there was no loss to follow-up.

Table 1 summarizes the clinical characteristics of the entire study population and of the 2 groups divided according to the median of ISWT (Table 1). The patients were significantly younger in long-ISWT group than short-ISWT group, and handgrip and quadriceps strength were higher in long-ISWT group as compared with those of short-ISWT group, otherwise the differences were not statistically significant. When compared between lowand high-handgrip-strength groups, the differences of sex, body weight, and SMI in addition to exercise param- 


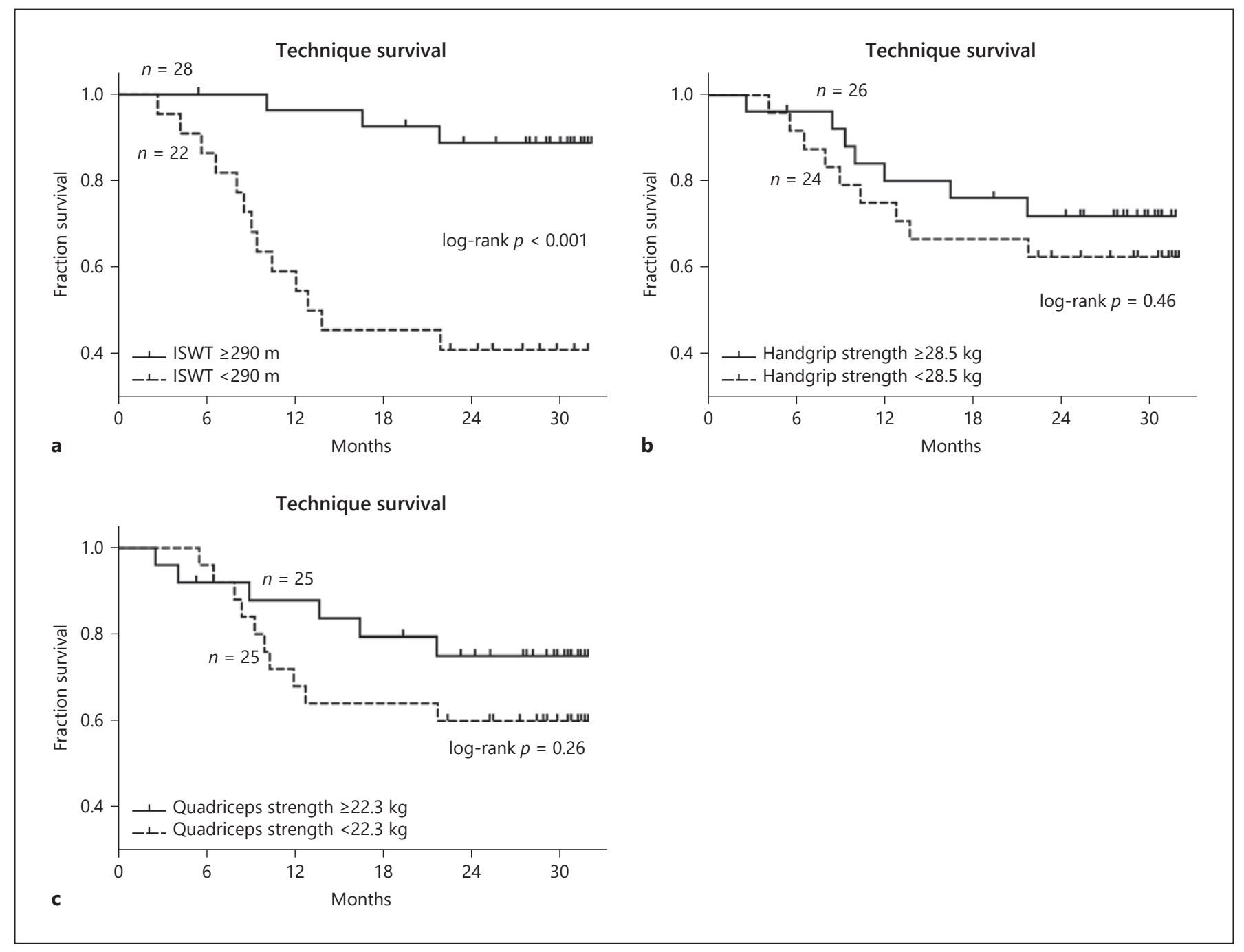

Fig. 2. Kaplan-Meier analysis of death- and transplant-censored technique survival rate for groups divided according to median ISWT (a), handgrip strength (b), and quadriceps strength (c). ISWT, incremental shuttle walking test.

eters were statistically significant (see online suppl. Table 1; see www.karger.com/doi/10.1159/000511293 for all online suppl. material), and when compared between low- and high-quadriceps-strength groups, the differences of sex, age, body weight, BMI, and SMI in addition to exercise parameters were statistically significant (online suppl. Table 2).

\section{Association between Exercise Capacity and Technique}

Survival

Three (11\%) of the 28 participants in the long-ISWT group and 13 (59\%) of the 22 in the short-ISWT group transferred to HD. Median technique survival time was significantly reduced in the short-ISWT group compared with the long-ISWT group (17.8 vs. 30.1 months, respectively; $p<0.001$ ) (Fig. 2a). Median technique survival time was not statistically different between the low- and high-handgrip-strength groups (23.8 vs. 26.2 months, respectively; $p=0.46$ ) (Fig. $2 \mathrm{~b}$ ) or low- and high-quadriceps-strength groups (23.4 and 26.9 months, respectively; $p=0.26$ ) (Fig. 2c). When participants were stratified according to the presence of assistance, median technique survival time was significantly higher among those undergoing assisted PD than those without assistance in the short-ISWT group (14.7 vs. 28.4 months, respectively; $p=0.02$ ), whereas they did not show any statistical differences in median technique survival time in the longISWT group (29.8 vs. 32.0 months, respectively; $p=0.39$ ) 
Table 2. Results of multivariate Cox regression analysis associated with technique failure

\begin{tabular}{|c|c|c|c|c|c|c|}
\hline \multirow[t]{2}{*}{ Independent variables } & \multicolumn{3}{|c|}{ Model 1} & \multicolumn{3}{|c|}{ Model 2} \\
\hline & HR & $95 \% \mathrm{CI}$ & $p$ value & $\mathrm{HR}$ & $95 \% \mathrm{CI}$ & $p$ value \\
\hline \multicolumn{7}{|l|}{ Cause-specific hazards } \\
\hline Age (per 10 years) & 0.87 & $0.39-1.93$ & 0.88 & - & - & - \\
\hline Assisted PD & 0.08 & $0.009-0.72$ & 0.02 & 0.06 & $0.007-0.52$ & 0.01 \\
\hline Frailty & 0.24 & $0.02-3.15$ & 0.28 & - & - & - \\
\hline CCI & 2.04 & $1.17-3.56$ & 0.01 & 1.90 & $1.20-3.00$ & 0.006 \\
\hline PD vintage (years) & 1.05 & $0.83-1.32$ & 0.69 & - & - & - \\
\hline GNRI (per 10) & 0.49 & $0.26-0.93$ & 0.03 & 0.58 & $0.34-0.99$ & 0.04 \\
\hline ISWT (per 100 m) & 0.45 & $0.26-0.78$ & 0.004 & 0.53 & $0.35-0.79$ & 0.002 \\
\hline \multicolumn{7}{|l|}{ Subdistribution hazards } \\
\hline Age (per 10 years) & 0.87 & $0.33-2.28$ & 0.78 & - & - & - \\
\hline Assisted PD & 0.08 & $0.01-0.63$ & 0.02 & 0.11 & $0.02-0.77$ & 0.03 \\
\hline Frailty & 0.24 & $0.04-1.41$ & 0.11 & - & - & - \\
\hline CCI & 2.04 & $1.35-3.10$ & 0.001 & 1.56 & $1.05-2.39$ & 0.03 \\
\hline $\mathrm{PD}$ vintage (year) & 1.05 & $0.86-1.27$ & 0.63 & - & - & - \\
\hline GNRI (per 10) & 0.49 & $0.25-0.97$ & 0.04 & - & - & - \\
\hline ISWT (per $100 \mathrm{~m}$ ) & 0.45 & $0.24-0.83$ & 0.01 & 0.52 & $0.33-0.82$ & 0.005 \\
\hline
\end{tabular}

$\mathrm{HR}$, hazard ratio; CI, confidence interval; PD, peritoneal dialysis; GNRI, geriatric nutritional risk index; ISWT, incremental shuttle walking test; CCI, Charlson Comorbidity Index.

(online suppl. Fig. 1). In the sensitivity analysis of technique survival regarding death and transplantation as technique failure, the results were comparable with the main analysis (online suppl. Fig. 2). Cox univariate analysis revealed that shorter ISWT distance, presence of DM, history of cardiovascular disease, high CCI, and high serum phosphorus levels were risk factors of technique failure, whereas PD vintage and handgrip and quadriceps strength were not associated with technique failure. In multivariate analysis, the cause-specific hazard model used for standard Cox regression analysis revealed that lower ISWT, higher CCI, absence of assisted PD, and lower GNRI, which were independently associated with technique failure, remained as significant predictors of technique failure after stepwise backward elimination, whereas PD vintage had no significant association with technique failure (Table 2). These models were assumed to hold a proportional hazard assumption and had a sufficiently high concordance index of over 0.85 . The subdistribution hazard regression model showed a similar result, with elimination of GNRI in stepwise backward analysis.

\section{Influence of Exercise Capacity on Outcomes}

Peritonitis-free survival time was significantly shorter in the short-ISWT group than the long-ISWT group (20.3 and 27.2 months, respectively; $p=0.01$ ) (Fig. 3a). The peritonitis rates of the short- and long-ISWT groups were 0.32 and 0.08 per patient-year, respectively, with an overall rate of 0.16 per patient-year. Among participants who experienced peritonitis, 7 (70\%) out of 10 in the shortISWT group and 2 (40\%) out of 5 in the long-ISWT group ceased PD and transferred to HD or died due to peritonitis $(p=0.33)$. Peritonitis-free survival time was not statistically different between the low- and high-handgrip or quadriceps strength groups, either $(p=0.63$ and $p=0.72$, respectively) (Fig. 3b, c). The incidence of PD-related hospitalization was reported for 38 participants $(76 \%)$, and causes of 1st hospitalization within the study period included peritonitis $(n=10)$, uncontrolled overhydration $(n=10)$, uremic solute retention $(n=5)$, tunnel infection $(n=4)$, decreased levels of daily activity $(n=3)$, severe anemia $(n=3)$, catheter malfunction $(n=2)$, and volume depletion $(n=1)$. The PD-related hospitalization-free survival time was significantly shorter in the short-ISWT group than the long-ISWT group (9.7 vs. 17.4 months, respectively; $p=0.003$ ) (Fig. $4 \mathrm{a}$ ). On the contrary, PDrelated hospitalization-free survival time was not statistically different between low- and high-handgrip or quadriceps strength groups ( $p=0.46$ and $p=0.84$, respectively) (Fig. 4b, c). 


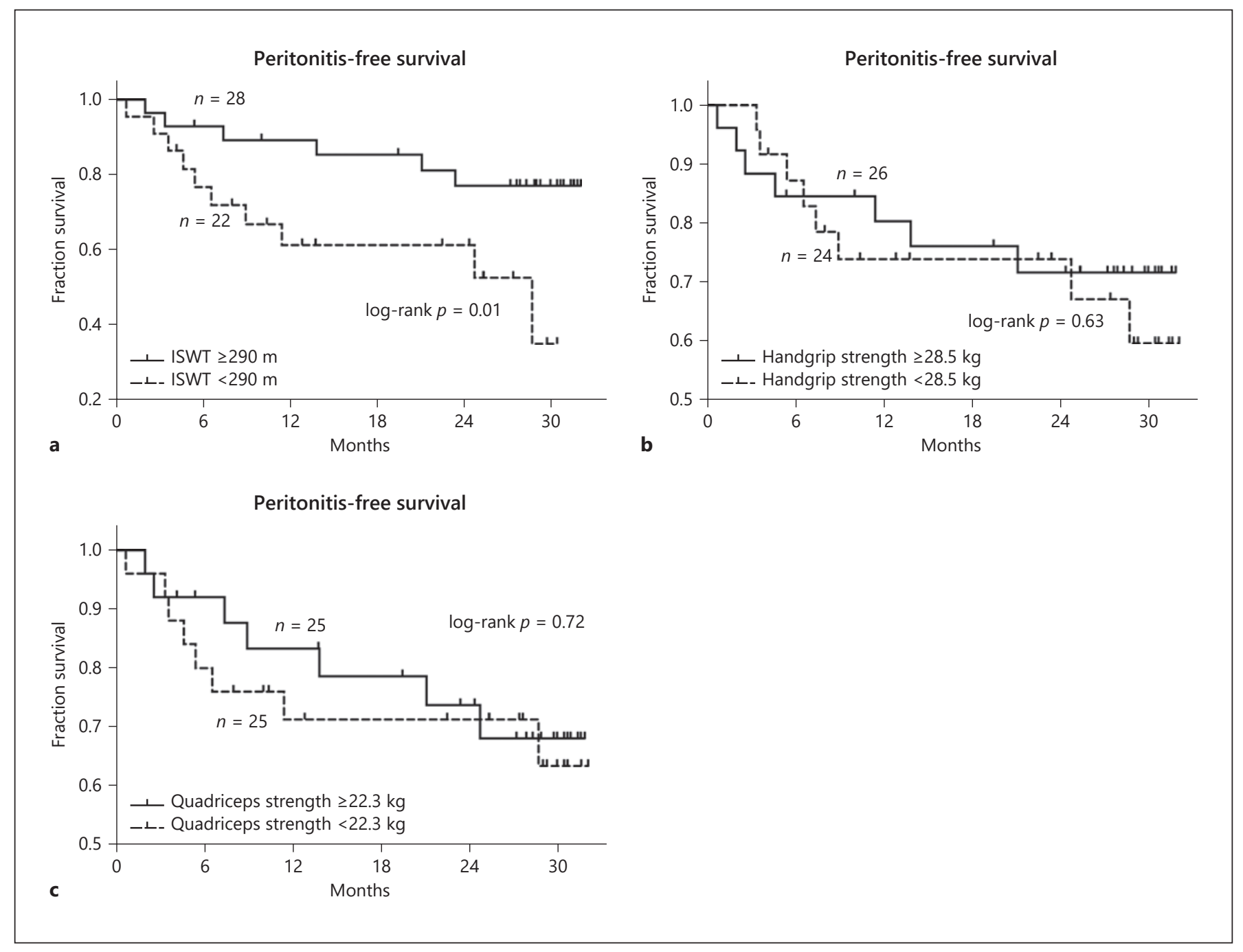

Fig. 3. Kaplan-Meier analysis of peritonitis-free survival rate for groups divided according to median ISWT (a), handgrip strength (b), and quadriceps strength (c). ISWT, incremental shuttle walking test.

\section{Discussion/Conclusion}

Although age, cause of end-stage kidney disease, DM, $\mathrm{CCI}$, center size, PD modality, and the PD system have been reported to be factors predicting technique survival in PD therapy, there have been few studies focusing on the association of exercise capacity with technique survival [13-15]. Our results demonstrate that, in a cohort of patients on maintenance PD, ISWT is an exercise parameter, which is an independent factor predicting technique survival. This result persisted even after adjusting for age, DM, use of assisted PD, frailty, CCI, PD vintage, urine output, and GNRI. In contrast, no difference was observed in technique survival between groups divided according to handgrip or quadriceps strength. This seems to be attributable that ISWT was also reflecting cardiorespiratory capacity plus muscular strength. The long-ISWT group also exhibited improved rates of peritonitis-free survival and PD-related hospitalization-free survival. As peritonitis or overhydration are primary reasons for the cessation of PD therapy, it is reasonable to conclude that these factors contributed to the increased technique survival observed in the long-ISWT group [18].

To the best of our knowledge, Shi et al. [7] only reported the association of an exercise parameter with technique survival, using 6-min walking test. In the present study, we demonstrated it with ISWT. Compared with 6-min walking test, ISWT has a higher repro- 


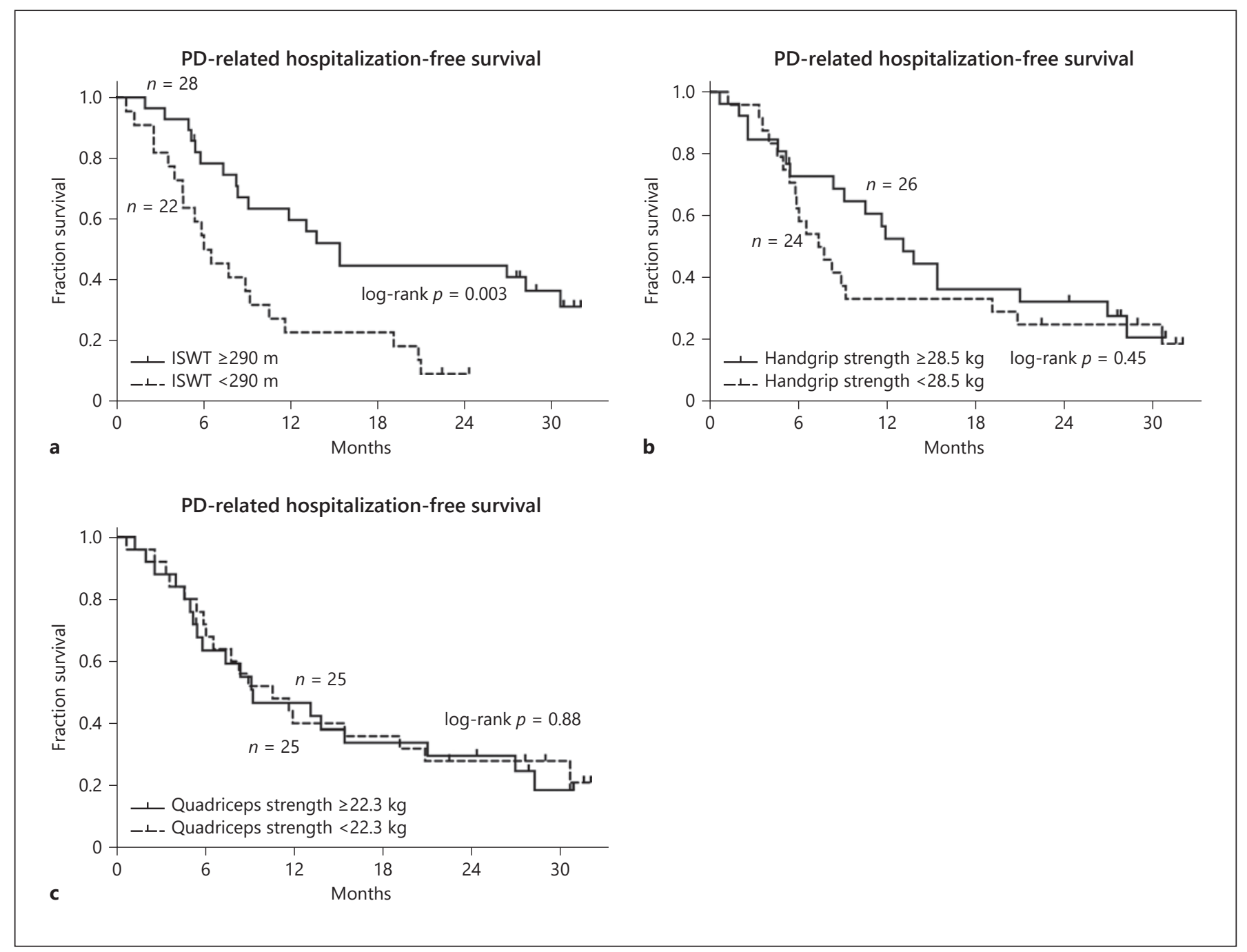

Fig. 4. Kaplan-Meier analysis of peritoneal-dialysis-related-hospitalization-free survival rate for groups divided according to median ISWT (a), handgrip strength (b), and quadriceps strength (c). ISWT, incremental shuttle walking test; PD, peritoneal dialysis.

ducibility and furthermore can be conveniently used for an exercise prescription according to estimated peak oxygen uptake $[19,20]$. A previous study demonstrated that a 12-week home-based exercise program based on ISWT can improve the aerobic capacity of PD patients without any adverse effects [21]. This finding suggests that home-based exercise therapy on ISWT has the potential to counter the higher technique failure rate associated with PD than HD. Verification of this hypothesis is one of the goals of future interventional studies, in the situation that there is still a distinct lack of knowledge of exercise therapy in patients undergoing PD compared with those undergoing HD; studies have shown that exercise capacity is associated with mortality, cardiovascu- lar function, and health-related quality of life, which can be improved by exercise training in patients on HD [2226].

Presently, the phenomenon of rapid population aging is observed in all developed countries; thus, assisted PD, which enables elderly patients to receive $\mathrm{PD}$ at home, is attracting a lot of attention. In France, over $50 \%$ of patients on PD aged 75 years or over receive assisted PD [27]. The increased technique survival rate of the shortISWT group upon assisted PD implies that assisted PD might be beneficial not only for elderly patients but also for those with low exercise tolerance. Additionally, among the 8 patients who were excluded due to physical complications unsuitable for exercise, all patients receiving as- 
sisted PD continued PD until death, while those undergoing self-care $\mathrm{PD}$ were transferred to HD within approximately a year, suggesting that even in patients with severe conditions, assisted PD might be helpful in preventing unplanned transfer to HD. In order to make effective use of the limited medical welfare service such as home-visit nurse care, which is necessary for assisted PD, it needs to be revealed what kind of patients should preferentially receive assisted PD.

Although the reason why only ISWT distance among exercise parameters was associated with technique survival is unclear, we proposed a multifaceted hypothesis. One possible explanation is the correlation between ISWT and physical activity in daily life. It has been reported that ISWT is significantly associated with physical activity in PD patients compared with handgrip and quadriceps strength $[8,28]$. Physical activity affects muscle contraction-induced myokine secretion, which has a lot of physiological and clinical effects including antiinflammatory or antidepressant action [29]. This might be a factor that only ISWT among exercise parameters could predict technique survival. The other possible explanation is possibility of cognitive function as a confounding factor. Cognitive impairment is associated with technique failure. Walking requires the comprehensive ability including attention, executive and visuospatial function, and the motor processing functions of the motor cortex, basal ganglia, and cerebellum [30]. Otobe et al. [31] demonstrated that solely gait speed was significantly associated with cognitive impairment among several exercise parameters including handgrip and leg strength in CKD patients. Although cognitive function could not be examined in the present study, it is possible that the long-ISWT group had better cognitive function than the short-ISWT group, which partially contributed to the difference in technique failure. The final explanation is the association of ISWT with healthrelated quality of life (HRQOL). We previously demonstrated that not handgrip and quadriceps strength but only ISWT was strongly correlated with higher HRQOL subscales [8]. It was suggested that better HRQOL lead to better technical and patient survival rates in the previous study [32]; therefore, higher ISWT might reflect better HRQOL, which partly contributed to the improvement of technical survival.

The present study has several limitations which should be acknowledged. First, this was an observational cohort study, which makes it difficult to prove causality between ISWT and outcomes in patients on PD. In addition, because this study was performed in a single center and the sample size was relatively small, we have to use stepwise backward elimination in multivariate regression analysis. However, our model had enough concordance index for a goodness of fit. Finally, our present study also excluded patients who could not perform the ISWT; therefore, we could not examine the association of PD technique survival with excise capacity in such patients, probably including the patients with severe frailty. The exclusion criteria might have contributed to an important selection bias, which should be considered while interpreting the results. However, considering that the presence of frailty as a binary parameter was not associated with technique failure in both univariate and multivariate analysis, we could conclude that ISWT as a continuous variable was a sensitive and useful parameter predicting technique failure in general patients on PD. Our results should be interrupted cautiously owing to possible selection bias and subsequent statistical analysis.

In conclusion, we demonstrate that ISWT is an important predictor of technique survival in patients undergoing maintenance PD. The introduction of assisted PD to patients with low exercise tolerance can be beneficial in terms of technique survival. In addition, our results should be interrupted cautiously owing to the large number of limitations, possible selection bias, and subsequent statistical analysis. Further intervention studies are needed to verify whether exercise therapy can improve PD technique survival and whether assisted PD can improve the technique survival of patients with low exercise tolerance.

\section{Statement of Ethics}

This study and all its protocols were reviewed and approved by the Ethics Committee of our hospital, and written informed consent was obtained from all patients prior to participation.

\section{Conflict of Interest Statement}

The authors have no conflicts of interest to declare.

\section{Funding Sources}

The authors did not receive any funding. 


\section{Author Contributions}

T.N. and K.U. designed the study and wrote the initial draft of the manuscript. K.U., N.W., K. Morimoto, K. Muraoka, and K.A. contributed to data collection. T.N., K.U., K. Muraoka, K. Miyashita, and S.W. contributed to analysis and interpretation of data and assisted in the preparation of the manuscript. H.I. supervised the manuscript. All authors approved the final version of the manuscript and agree to be accountable for all aspects of the work in ensuring that questions related to the accuracy or integrity of any part of the work are appropriately investigated and resolved.

\section{References}

1 Chang YT, Hwang JS, Hung SY, Tsai MS, Wu JL, Sung JM, et al. Cost-effectiveness of hemodialysis and peritoneal dialysis: a national cohort study with 14 years follow-up and matched for comorbidities and propensity score. Sci Rep. 2016;6:30266.

2 Mehrotra R, Devuyst O, Davies SJ, Johnson DW. The current state of peritoneal dialysis. J Am Soc Nephrol. 2016;27(11):3238-52.

3 Li PK, Chow KM, Van de Luijtgaarden MW, Johnson DW, Jager KJ, Mehrotra R, et al. Changes in the worldwide epidemiology of peritoneal dialysis. Nat Rev Nephrol. 2017; 13(2):90-103.

4 Fahal IH. Uraemic sarcopenia: aetiology and implications. Nephrol Dial Transplant. 2014; 29(9):1655-65.

5 Pereira RA, Cordeiro AC, Avesani CM, Carrero JJ, Lindholm B, Amparo FC, et al. Sarcopenia in chronic kidney disease on conservative therapy: prevalence and association with mortality. Nephrol Dial Transplant. 2015; 30(10):1718-25.

6 Kamijo Y, Kanda E, Ishibashi Y, Yoshida M. Sarcopenia and frailty in PD: impact on mortality, malnutrition, and inflammation. Perit Dial Int. 2018;38(6):447-54.

7 Shi Y, Zheng D, Zhang L, Yu Z, Yan H, Ni Z, et al. Six-minute walk test predicts all-cause mortality and technique failure in ambulatory peritoneal dialysis patients. Nephrology. 2017;22(2):118-24.

8 Uchiyama K, Washida N, Muraoka K, Morimoto K, Kasai T, Yamaki K, et al. Exercise capacity and association with quality of life in peritoneal cialysis patients. Perit Dial Int. 2019;39(1):66-73.

9 American College of Sports Medicine. ACSM's guidelines for exercise testing and prescription. 10th ed.; 2016.

10 JCS Joint Working Group. Guidelines for rehabilitation in patients with cardiovascular disease (JCS 2012). Circ J. 2014;78(8):202293.

11 Kang SH, Cho KH, Park JW, Yoon KW, Do JY. Geriatric nutritional risk index as a prognostic factor in peritoneal dialysis patients. Perit Dial Int. 2013;33(4):405-10.

12 Figueiredo A, Goh BL, Jenkins S, Johnson DW, Mactier R, Ramalakshmi S, et al. Clinical practice guidelines for peritoneal access. Perit Dial Int. 2010;30(4):424-9.
13 Chidambaram M, Bargman JM, Quinn RR, Austin PC, Hux JE, Laupacis A. Patient and physician predictors of peritoneal dialysis technique failure: a population based, retrospective cohort study. Perit Dial Int. 2011; 31(5):565-73.

14 See EJ, Johnson DW, Hawley CM, Pascoe EM, Badve SV, Boudville N, et al. Risk predictors and causes of technique failure within the first year of peritoneal dialysis: an Australia and New Zealand Dialysis and Transplant Registry (ANZDATA) study. Am J Kidney Dis. 2018;72(2):188-97.

15 Boudville N, Ullah S, Clayton P, Sud K, Borlace $\mathrm{M}, \mathrm{Badve} S \mathrm{~V}$, et al. Differences in peritoneal dialysis technique survival between patients treated with peritoneal dialysis systems from different companies. Nephrol Dial Transplant. 2019;34(6):1035-44.

16 Lau B, Cole SR, Gange SJ. Competing risk regression models for epidemiologic data. Am J Epidemiol. 2009;170(2):244-56.

17 Teixeira L, Rodrigues A, Carvalho MJ, Cabrita A, Mendonça D. Modelling competing risks in nephrology research: an example in peritoneal dialysis. BMC Nephrol. 2013;14: 110.

18 Nakayama M, Miyazaki M, Honda K, Kasai K, Tomo T, Nakamoto H, et al. Encapsulating peritoneal sclerosis in the era of a multi-disciplinary approach based on biocompatible solutions: the NEXT-PD study. Perit Dial Int. 2014;34(7):766-74.

19 Saglam M, Vardar-Yagli N, Savci S, Inal-Ince D, Aribas Z, Bosnak-Guclu M, et al. Six minute walk test versus incremental shuttle walk test in cystic fibrosis. Pediatr Int. 2016;58(9): 887-93.

20 Singh SJ, Morgan MD, Scott S, Walters D, Hardman AE. Development of a shuttle walking test of disability in patients with chronic airways obstruction. Thorax. 1992;47(12): 1019-24.

21 Uchiyama K, Washida N, Morimoto K, Muraoka K, Kasai T, Yamaki K, et al. Homebased aerobic exercise and resistance training in peritoneal dialysis patients: a randomized controlled trial. Sci Rep. 2019;9(1):2632.

22 Sietsema KE, Amato A, Adler SG, Brass EP. Exercise capacity as a predictor of survival among ambulatory patients with end-stage renal disease. Kidney Int. 2004;65(2):719-24.
23 Tentori F, Elder SJ, Thumma J, Pisoni RL, Bommer J, Fissell RB, et al. Physical exercise among participants in the Dialysis Outcomes and Practice Patterns Study (DOPPS): correlates and associated outcomes. Nephrol Dial Transplant. 2010;25(9):3050-62.

24 Ouzouni S, Kouidi E, Sioulis A, Grekas D, Deligiannis A. Effects of intradialytic exercise training on health-related quality of life indices in haemodialysis patients. Clin Rehabil. 2009;23(1):53-63.

25 de Lima MC, Cicotoste CL, Cardoso KS, Forgiarini LA Jr, Monteiro MB, Dias AS. Effect of exercise performed during hemodialysis: strength versus aerobic. Ren Fail. 2013;35(5): 697-704.

26 Hristea D, Deschamps T, Paris A, Lefrançois G, Collet V, Savoiu C, et al. Combining intradialytic exercise and nutritional supplementation in malnourished older haemodialysis patients: towards better quality of life and autonomy. Nephrology. 2016;21(9):785-90.

27 Castrale C, Evans D, Verger C, Fabre E, Aguilera D, Ryckelynck JP, et al. Peritoneal dialysis in elderly patients: report from the French Peritoneal Dialysis Registry (RDPLF). Nephrol Dial Transplant. 2010;25(1):255-62.

28 Painter PL, Agarwal A, Drummond M. Physical function and physical activity in peritoneal dialysis patients. Perit Dial Int. 2017; 37(6):598-604.

29 Fiuza-Luces C, Garatachea N, Berger NA, Lucia A. Exercise is the real polypill. Physiology. 2013;28(5):330-58.

30 Buracchio T, Dodge HH, Howieson D, Wasserman D, Kaye J. The trajectory of gait speed preceding mild cognitive impairment. Arch Neurol. 2010;67(8):980-6.

31 Otobe Y, Hiraki K, Hotta C, Nishizawa H, Izawa KP, Taki Y, et al. Mild cognitive impairment in older adults with pre-dialysis patients with chronic kidney disease: Prevalence and association with physical function. Nephrology. 2019;24(1):50-5.

32 Paniagua R, Amato D, Vonesh E, Guo A, Mujais S, Mexican Nephrology Collaborative Study Group. Health-related quality of life predicts outcomes but is not affected by peritoneal clearance: the ADEMEX trial. Kidney Int. 2005;67(3):1093-104. 